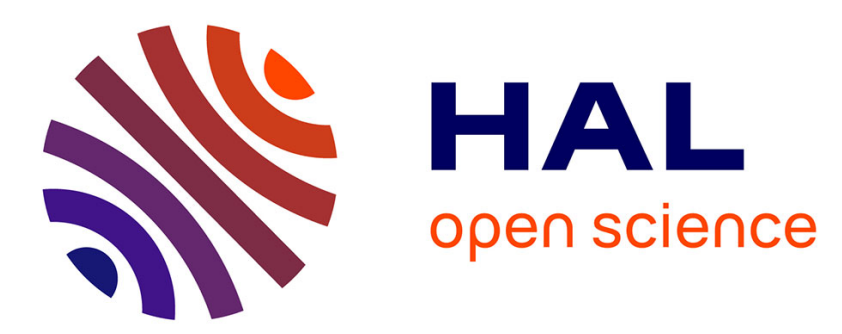

\title{
A conservative scheme for non-classical solutions to a strongly coupled PDE-ODE problem
}

Christophe Chalons, Maria Laura Delle Monache, Paola Goatin

\section{To cite this version:}

Christophe Chalons, Maria Laura Delle Monache, Paola Goatin. A conservative scheme for nonclassical solutions to a strongly coupled PDE-ODE problem. Interfaces and Free Boundaries, 2017, 19 (4), pp.553-570. 10.4171/IFB/392 . hal-01070262

\section{HAL Id: hal-01070262 \\ https://hal.inria.fr/hal-01070262}

Submitted on 30 Sep 2014

HAL is a multi-disciplinary open access archive for the deposit and dissemination of scientific research documents, whether they are published or not. The documents may come from teaching and research institutions in France or abroad, or from public or private research centers.
L'archive ouverte pluridisciplinaire HAL, est destinée au dépôt et à la diffusion de documents scientifiques de niveau recherche, publiés ou non, émanant des établissements d'enseignement et de recherche français ou étrangers, des laboratoires publics ou privés. 


\title{
A CONSERVATIVE SCHEME FOR NON-CLASSICAL SOLUTIONS TO A STRONGLY COUPLED PDE-ODE PROBLEM ${ }^{\dagger}$
}

\author{
C. CHALONS $\ddagger$, M.L. DELLE MONACHE §, AND P. GOATIN ף
}

\begin{abstract}
We consider a strongly coupled PDE-ODE system that describes the influence of a slow and large vehicle on road traffic. The model consists of a scalar conservation law describing the main traffic evolution and an ODE accounting for the trajectory of the slower vehicle that depends on the downstream traffic density. The moving constraint is operated by an inequality on the flux, which accounts for the bottleneck created on the road by the presence of the slower vehicle. We introduce a conservative scheme for the constrained hyperbolic PDE and a tracking algorithm for the ODE. We show numerical tests and compute numerically the order of convergence.
\end{abstract}

Key words. Scalar conservation laws with local moving constraints, Traffic flow modeling, PDE-ODE coupling, Conservative finite volume schemes.

35L65, 90B20.

1. Introduction We consider a coupled PDE-ODE model describing a large, slow moving vehicle (that we will refer to as "the bus") interacting with the sourrounding traffic flow. The system consists of the Lighthill-Whitham-Richards [18, 19] scalar conservation law, which describes the evolution in time of the mean traffic density on a road, and by an ODE accounting for the slower vehicle trajectory. The loss of road capacity due to the presence of the bus is expressed by an inequality constraint on the vehicles flow at the bus position.

The model was first introduced and studied in an engineering setting $[13,17]$ (see also $[7,8]$ for a numerical treatment). A rigorous mathematical framework and an existence result were later proposed in [10]. Other systems modeling moving bottlenecks on roads are proposed in the applied mathematics literature: in [12, 16] the flux constraint is modeled by a mollifier, thus preserving the smoothness of the flux function; a $2 \times 2$ system modeling traffic flow coupled with a second order ODE is presented in [2]. In these works, standard numerical methods can be applied.

Conservation laws with locally constrained flux function were first introduced in [6] to model fixed constraints like toll gates. Well-posedness results in the BV and $\mathbf{L}^{\infty}$ settings, as well as the convergence of adapted finite volume schemes are given in $[1,6]$. Extensions to non-concave fluxes and systems were also proposed in [5] and [11] respectively. In those papers, anyway, the constraint position is not affected by the solution of the conservation law, and the analytical and numerical treatments are therefore easier than in the present case. In particular, no stability result with respect to initial data is currently available for the PDE-ODE system considered in this paper. A first numerical strategy to compute approximate solutions was proposed in [9], based on locally non-uniform moving meshes to track the bus position.

In this paper we follow another approach to avoid non-uniform moving meshes. The main point here is related to the presence of non-classical shocks in the solutions of the model under consideration. It is well-known that, in this context, standard

*This research was partially supported by the European Research Council under the European Union's Seventh Framework Program (FP/2007-2013) / ERC Grant Agreement n. 257661.

$\dagger$ Received date / Revised version date

$\ddagger$ Université Versailles Saint-Quentin-en-Yvelines, (christophe.chalons@uvsq.fr).

§Inria Sophia Antipolis-Méditerranée, (maria-laura.delle_monache@inria.fr).

ฯInria Sophia Antipolis-Méditerranée, (paola.goatin@inria.fr). 
conservative finite volume methods cannot be applied and fail in producing good numerical results. Glimm's scheme can be used but it is not strictly conservative. In order to propose a numerical scheme which is conservative on fixed meshes and able to compute non-classical solutions, we propose to adapt to the present context of strongly coupled PDE-ODE system a reconstruction strategy approach introduced in [3], which allows to precisely capture moving non-classical discontinuities on fixed meshes still guaranteeing conservation, unlike Glimm's scheme. An important feature of the proposed method is to be exact for isolated classical and non-classical shocks, which means in particular only one point of numerical diffusion (on each cell the approximate value corresponds to the value of the average of the exact solution). In the general case, shocks are still computed without numerical diffusion and convergence is proved numerically. So far, however, no rigorous convergence result is available. Several test cases, some of them being significant from the modeling point of view, are proposed to illustrate these features.

2. Mathematical model The coupled PDE-ODE model in [10] reads

$$
\begin{array}{ll}
\partial_{t} \rho+\partial_{x} f(\rho)=0, & (t, x) \in \mathbb{R}^{+} \times \mathbb{R}, \\
\rho(0, x)=\rho_{0}(x), & x \in \mathbb{R}, \\
f(\rho(t, y(t)))-\dot{y}(t) \rho(t, y(t)) \leq \frac{\alpha R}{4 V}(V-\dot{y}(t))^{2} & t \in \mathbb{R}^{+}, \\
\dot{y}(t)=\omega(\rho(t, y(t)+)), & t \in \mathbb{R}^{+}, \\
y(0)=y_{0}, &
\end{array}
$$

where $\omega(\rho(t, y(t)+))$ denotes the right-hand limit of $\omega(\rho(t, x))$ at $y(t)$. Above, $\rho=$ $\rho(t, x) \in[0, R]$ is the scalar conserved quantity and represents the traffic density, whose maximum attainable value is $R$. The flux function $f:[0, R] \rightarrow \mathbb{R}^{+}$is assumed to be strictly concave and such that $f(0)=f(R)=0$. In this paper, we will take $f(\rho)=\rho v(\rho)$, where $v(\rho)=V(1-\rho / R)$ is the mean traffic speed, $V$ being the maximal velocity allowed on the road. The time-dependent variable $y$ denotes the bus position. If the traffic is not too congested, the bus moves at its own maximal speed $V_{b}<V$. When the surrounding traffic density is too high, the bus adapts its velocity accordingly. In particular, it is not possible for the bus to overtake the cars. From a mathematical point of view, the velocity of the bus can be described by the following traffic density dependent function (see Figure 2.1)

$$
\omega(\rho)= \begin{cases}V_{b} & \text { if } \rho \leq \rho^{*} \doteq R\left(1-V_{b} / V\right) \\ v(\rho) \text { otherwise }\end{cases}
$$

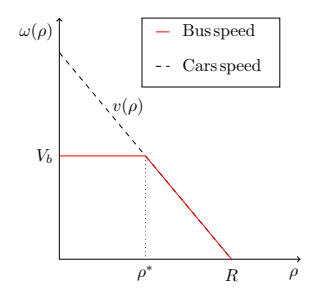

Fig. 2.1: Bus and cars speed. 
The bus acts as a moving obstacle, thus hindering the traffic flow as expressed by the flux constraint (2.1c). There, $\alpha \in] 0,1$ [ is the reduction rate of the road capacity due to the presence of the bus. The inequality (2.1c) is derived by studying the problem in the bus reference frame, i.e., setting $X=x-y(t)$ and rewriting the conservation law (2.1a) as

$$
\partial_{t} \rho+\partial_{X} F(\rho)=0, \quad F(\rho)=f(\rho)-\dot{y} \rho .
$$

In fact, let $f_{\alpha}:[0, \alpha R] \rightarrow \mathbb{R}^{+}$be the rescaled flux function describing the reduced flow at $x=y(t)$, i.e. $f_{\alpha}(\rho)=V \rho\left(1-\frac{\rho}{\alpha R}\right)$, and $\left.\rho_{\alpha} \in\right] 0, \alpha R / 2\left[\operatorname{such}\right.$ that $F_{\alpha}^{\prime}\left(\rho_{\alpha}\right)=0 \Leftrightarrow f_{\alpha}^{\prime}\left(\rho_{\alpha}\right)=$ $\dot{y}$ with $F_{\alpha}(\rho)=f_{\alpha}(\rho)-\dot{y} \rho$, i.e. $\rho_{\alpha}=\frac{\alpha R}{2}\left(1-\frac{\dot{y}}{V}\right)$, see Figure 2.2. Then observing that $F_{\alpha}=F_{\alpha}\left(\rho_{\alpha}\right)=f_{\alpha}\left(\rho_{\alpha}\right)-\dot{y} \rho_{\alpha}=\frac{\alpha R}{4 V}(V-\dot{y}(t))^{2}$, and imposing that in the bus reference frame the flux $F$ should be less than the maximum value of the flux of the reduced flow, at the bus position one gets (2.1c). Note that the inequality is always satisfied if $\dot{y}(t)=v(\rho)$, since the left-hand side is 0. Moreover, it is well defined even if $\rho$ has a jump at $y(t)$ because of the Rankine-Hugoniot conditions.

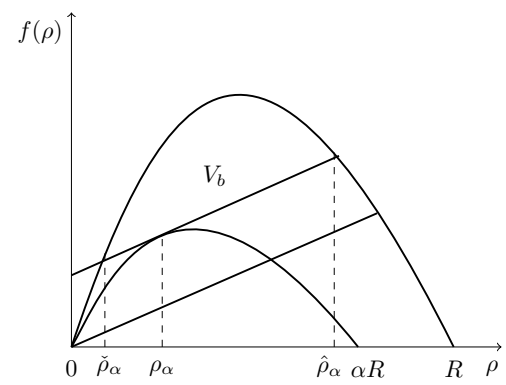

(a) Fixed reference frame

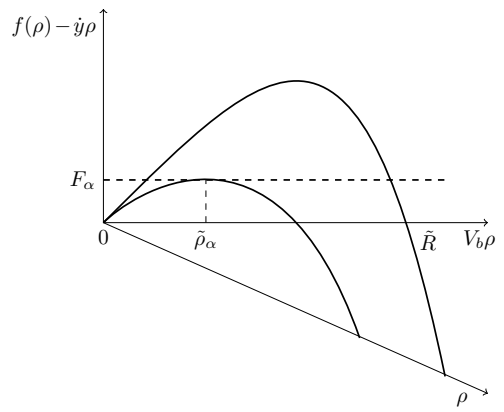

(b) Bus reference frame

Fig. 2.2: Flux functions for $\dot{y}=V_{b}$.

2.1. The Riemann Problem We recall here the definition of the Riemann Solver for problem (2.1) given in [10]. Let us consider a Riemann type initial datum

$$
\rho_{0}(x)=\left\{\begin{array}{l}
\rho_{L} \text { if } x<0, \\
\rho_{R} \text { if } x>0,
\end{array} \quad y_{0}=0\right.
$$

Denote by $\mathcal{R}$ the standard (i.e., without the constraint (2.1c)) Riemann solver for (2.1a)-(2.1b)-(2.4), i.e., the (right continuous) map $(t, x) \mapsto \mathcal{R}\left(\rho_{L}, \rho_{R}\right)(x / t)$ given by the standard weak entropy solution. Moreover, let $\check{\rho}_{\alpha}$ and $\hat{\rho}_{\alpha}$, with $\check{\rho}_{\alpha} \leq \hat{\rho}_{\alpha}$, be the intersection points of the flux function $f(\rho)$ with the line $f_{\alpha}\left(\rho_{\alpha}\right)+V_{b}\left(\rho-\rho_{\alpha}\right)$ (see Figure 2.2(a)).

Definition 2.1. The constrained Riemann solver $\mathcal{R}^{\alpha}:[0, R]^{2} \rightarrow \mathbf{L}_{\text {loc }}^{1}(\mathbb{R} ;[0, R])$ is defined as follows. 
1. If $f\left(\mathcal{R}\left(\rho_{L}, \rho_{R}\right)\left(V_{b}\right)\right)>F_{\alpha}+V_{b} \mathcal{R}\left(\rho_{L}, \rho_{R}\right)\left(V_{b}\right)$, then

$$
\mathcal{R}^{\alpha}\left(\rho_{L}, \rho_{R}\right)(x / t)=\left\{\begin{array}{l}
\mathcal{R}\left(\rho_{L}, \hat{\rho}_{\alpha}\right)(x / t) \text { if } x<V_{b} t, \\
\mathcal{R}\left(\check{\rho}_{\alpha}, \rho_{R}\right)(x / t) \text { if } x \geq V_{b} t, \quad \text { and } \quad y(t)=V_{b} t .
\end{array}\right.
$$

2. If $V_{b} \mathcal{R}\left(\rho_{L}, \rho_{R}\right)\left(V_{b}\right) \leq f\left(\mathcal{R}\left(\rho_{L}, \rho_{R}\right)\left(V_{b}\right)\right) \leq F_{\alpha}+V_{b} \mathcal{R}\left(\rho_{L}, \rho_{R}\right)\left(V_{b}\right)$, then

$$
\mathcal{R}^{\alpha}\left(\rho_{L}, \rho_{R}\right)=\mathcal{R}\left(\rho_{L}, \rho_{R}\right) \quad \text { and } \quad y(t)=V_{b} t .
$$

3. If $f\left(\mathcal{R}\left(\rho_{L}, \rho_{R}\right)\left(V_{b}\right)\right)<V_{b} \mathcal{R}\left(\rho_{L}, \rho_{R}\right)\left(V_{b}\right)$, i.e., $v\left(\mathcal{R}\left(\rho_{L}, \rho_{R}\right)\left(V_{b}\right)\right)<V_{b}$ then

$$
\mathcal{R}^{\alpha}\left(\rho_{L}, \rho_{R}\right)=\mathcal{R}\left(\rho_{L}, \rho_{R}\right) \quad \text { and } \quad y(t)=v\left(\rho_{R}\right) t .
$$

Note that, when the constraint is enforced (point 1 . in the above definition), a non-classical shock arises between $\hat{\rho}_{\alpha}$ and $\check{\rho}_{\alpha}$, which satisfies the Rankine-Hugoniot condition but violates the Lax entropy condition.

The existence of solutions for the general Cauchy problem with BV initial data is proved in [10]. In particular, solutions to (2.1d)-(2.1e) are to be intended in the Carathéodory sense.

3. Numerical method Our aim is to design a scheme that is able to approximate numerically on a fixed mesh the non-classical shock and to track at the same time the bus position. Let $\Delta x$ and $\Delta t$ be the fixed space and time steps, and set $x_{j+1 / 2}=j \Delta x, x_{j}=(j-1 / 2) \Delta x$ for $j \in \mathbb{Z}$ and $t^{n}=n \Delta t$ for $n \in \mathbb{N}$.

3.1. Conservative scheme for hyperbolic PDEs with constraint To approximate the conservation law we use a conservative finite volume scheme. We approximate the initial datum by a piecewise constant function given by its average on the discretization cells $C_{j}=\left[x_{j-\frac{1}{2}}, x_{j+\frac{1}{2}}\left[, \rho_{j}^{0}=\frac{1}{\Delta x} \int_{x_{j-\frac{1}{2}}}^{x_{j+\frac{1}{2}}} \rho_{0}(x) d x, \quad j \in \mathbb{Z}\right.\right.$. To compute the approximation $\rho_{j}^{n}$ of the solution $\rho$ at time $t^{n}$ and on the cell $C_{j}$, for $j \in \mathbb{Z}$ and $n \geq 1$, we look for a recursive update procedure of the classical form

$$
\rho_{j}^{n+1}=\rho_{j}^{n}-\frac{\Delta t}{\Delta x}\left(F_{j+\frac{1}{2}}^{n}-F_{j-\frac{1}{2}}^{n}\right),
$$

where the numerical fluxes $F_{j+\frac{1}{2}}^{n}$ at the cell interfaces $x_{j+\frac{1}{2}}$ have to be suitably defined in order to capture non-classical shocks.

Following [3], we propose an algorithm that is able to recreate the discontinuity of the non-classical shock using a reconstruction technique. Indeed, whenever a nonclassical shock appears, the classical finite volume schemes, like Godunov's [14], fail to generate good approximations of the exact solution, see [3]. We proceed as follows. Assume that at time $t^{n}$, the bus position $y^{n}$ is located in the cell $C_{m}$ for a given $m$. Whenever condition

$$
f\left(\rho_{m}^{n}\right)>F_{\alpha}+V_{b} \rho_{m}^{n},
$$

is satisfied, we consider that a non-classical shock is expected to appear locally around the bus. Hence, we propose to introduce in the cell $C_{m}$ the left and right states $\rho_{m, l}^{n}=\hat{\rho}_{\alpha}$ and $\rho_{m, r}^{n}=\check{\rho}_{\alpha}$ of the non-classical shock, which is expected to be present in the Riemann solution associated with $\rho_{m-1}^{n}$ and $\rho_{m+1}^{n}$ in case also inequality

$$
f\left(\mathcal{R}\left(\rho_{m-1}^{n}, \rho_{m+1}^{n}\right)\left(V_{b}\right)\right)>F_{\alpha}+V_{b} \mathcal{R}\left(\rho_{m-1}^{n}, \rho_{m+1}^{n}\right)\left(V_{b}\right),
$$




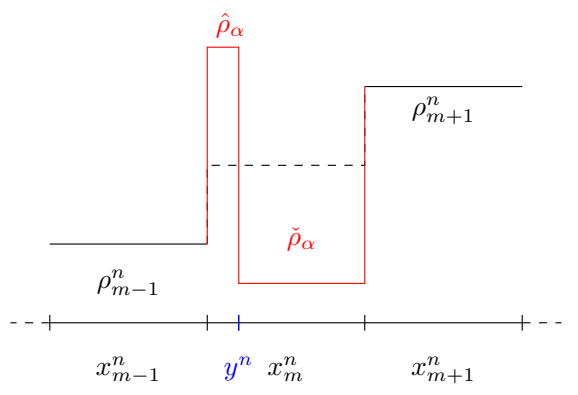

Fig. 3.1: Reconstruction of a non-classical shock.

is satisfied. Since the presence of the non-classical shock is due to the presence of the bus, we require this reconstructed discontinuity is located inside $C_{m}$ at a position

$$
\bar{x}_{m}=x_{m-\frac{1}{2}}+d_{m}^{n} \Delta x,
$$

for some $d^{m}$ defined such that the reconstruction procedure is conservative, which means

$$
d_{m}^{n} \rho_{m, l}^{n}+\left(1-d_{m}^{n}\right) \rho_{m, r}^{n}=\rho_{m}^{n}
$$

or equivalently,

$$
d_{m}^{n}=\frac{\rho_{m, r}^{n}-\rho_{m}^{n}}{\rho_{m, r}^{n}-\rho_{m, l}^{n}} .
$$

Clearly, it is possible to reconstruct the discontinuity provided that

$$
0 \leq d_{m}^{n} \leq 1
$$

To conclude the definition of the reconstruction strategy, let us mention that the non-classical discontinuity moves with speed $V_{b}>0$, so that a natural definition of the numerical flux at $x_{m+\frac{1}{2}}$ is given by

$$
\Delta t F_{m+\frac{1}{2}}^{n}=\min \left(\Delta t_{m+\frac{1}{2}}, \Delta t\right) f\left(\rho_{m, r}^{n}\right)+\max \left(\Delta t-\Delta t_{m+\frac{1}{2}}, 0\right) f\left(\rho_{m, l}^{n}\right)
$$

where $\Delta t_{m+\frac{1}{2}}=\frac{1-d_{m}^{n}}{V_{b}} \Delta x$ is the time needed by the discontinuity to reach the interface $x_{m+\frac{1}{2}}$. This definition of the flux is used provided that (3.2),(3.3), (3.7), (3.5), (3.6) are satisfied. Otherwise, and also for defining $F_{j+\frac{1}{2}}^{n}$ for $j \neq m$, a monotone and consistent numerical flux, like Godunov's, is used under the classical CFL condition:

$$
\Delta t \max _{j \in \mathbb{Z}}\left|f^{\prime}\left(\rho_{j}^{n}\right)\right| \leq \frac{1}{2} \Delta x .
$$

Following [3], it can be easily proved that isolated non-classical shocks are exactly captured with such a strategy (see also Case 0 in Section 4 below) and contain no numerical diffusion. Nevertheless, at this stage, classical shocks will suffer some numerical diffusion since classical Godunov's method is used away from the bus position, 
and this might be problematic in the validation of the solution on the constraint (3.3). In fact, if we choose initial data in the neighborhoods of the values $\hat{\rho}_{\alpha}, \check{\rho}_{\alpha}$, we observed that the approximate solution generated by this method may not be correct. To overcome this problem, we apply the reconstruction technique also to classical shocks. Numerical diffusion is thus avoided also for classical shocks and we get correct solutions. In the spirit of [15], we proceed as follows: given a cell $C_{j}$ for some $j \in \mathbb{Z}, j \neq m$, such that $\rho_{j-1}^{n}<\rho_{j+1}^{n}$, we introduce the left and right traces $\rho_{l}^{n}=\rho_{j-1}^{n}$ and $\rho_{r}^{n}=\rho_{j+1}^{n}$ of a reconstructed discontinuity and we define $d_{j}^{n}$, by

$$
d_{j}^{n}=\frac{\rho_{r}^{n}-\rho_{j}^{n}}{\rho_{r}^{n}-\rho_{l}^{n}} .
$$

Let us denote by $\lambda\left(\rho_{l}^{n}, \rho_{r}^{n}\right)$ the speed of the discontinuity given by the RankineHugoniot condition, i.e.,

$$
\lambda\left(\rho_{l}^{n}, \rho_{r}^{n}\right)=\frac{f\left(\rho_{l}^{n}\right)-f\left(\rho_{r}^{n}\right)}{\rho_{l}^{n}-\rho_{r}^{n}} .
$$

Then, if it is actually possible to reconstruct such a discontinuity within the cell $C_{j}$, that is to say if $d_{j}^{n} \in[0,1]$, the numerical fluxes at $x_{j \pm \frac{1}{2}}$ are defined by

- if $\lambda\left(\rho_{l}^{n}, \rho_{r}^{n}\right) \geq 0$,

$$
\Delta t F_{j+\frac{1}{2}}^{n}=\min \left(\Delta t_{j+\frac{1}{2}}, \Delta t\right) f\left(\rho_{r}^{n}\right)+\max \left(\Delta t-\Delta t_{j+\frac{1}{2}}, 0\right) f\left(\rho_{l}^{n}\right),
$$

with $\Delta t_{j+\frac{1}{2}}=\frac{1-d_{j}^{n}}{\lambda\left(\rho_{l}^{n}, \rho_{r}^{n}\right)} \Delta x$.

- if $\lambda\left(\rho_{l}^{n}, \rho_{r}^{n}\right) \leq 0$,

$$
\Delta t F_{j-\frac{1}{2}}^{n}=\min \left(\Delta t_{j-\frac{1}{2}}, \Delta t\right) f\left(\rho_{l}^{n}\right)+\max \left(\Delta t-\Delta t_{j-\frac{1}{2}}, 0\right) f\left(\rho_{r}^{n}\right),
$$

with $\Delta t_{j-\frac{1}{2}}=\frac{d_{j}^{n}}{-\lambda\left(\rho_{l}^{n}, \rho_{r}^{n}\right)} \Delta x$

where, with some abuse of notation, we mean that if $\lambda\left(\rho_{l}^{n}, \rho_{r}^{n}\right)=0$ then $F_{j+\frac{1}{2}}^{n}=f\left(\rho_{r}^{n}\right)$ and $F_{j-\frac{1}{2}}^{n}=f\left(\rho_{l}^{n}\right)$. This additional reconstruction guarantees that also isolated classical shocks are exactly captured, as shown by the numerical tests presented in Section 4 . Moreover, with this supplementary reconstruction we are able to handle correctly all the interactions among shocks, classical and non-classical.

REMARK 3.1. In the event of an ambiguity, for instance $j=m+1$ and $\lambda \leq 0$ above, the non-classical discontinuity reconstruction is always preferred.

3.2. Numerical method for the ODE To precisely track the bus trajectory we adapt the algorithm introduced in [4]. At each time step, we update the position $y^{n}$ of the bus by studying interactions between the bus trajectory and the density waves within the corresponding cell. We distinguish two cases:

- Inequality (3.2) is satisfied. Then the bus moves at velocity $V_{b}$ and we update the bus position as $y^{n+1}=V_{b} \Delta t^{n}+y^{n}$.

- Condition (3.2) is not satisfied. In this case the solution is classical and we implement the tracking algorithm introduced in [4]. We have to distinguish two situations: one when $y^{n} \in\left[x_{m-\frac{1}{2}}, x_{j}^{n}\left[\right.\right.$ and one when $y^{n} \in\left[x_{j}^{n}, x_{m+\frac{1}{2}}[\right.$. In both cases, we check if the wave starting at the cell interface is a shock or a rarefaction and compute the time of interaction between the wave and the bus trajectory.

The cell index $m$ is updated according to the new position of the bus. 
4. Numerical results In this section we present some numerical tests performed with the scheme previously described. We deal with a road of length 1 parameterized by the interval $[0,1]$. In all the simulations we fix $V_{b}=0.3, \alpha=0.6, V=R=1$. Case 0: We consider Riemann type initial data with

$$
\rho_{0}(x)=\left\{\begin{array}{ll}
\hat{\rho}_{\alpha} & \text { if } x<0.5, \\
\check{\rho}_{\alpha} & \text { if } x>0.5,
\end{array} \quad y_{0}=0.5\right.
$$

The solution is given by a non-classical shock, as illustrated in Figure 4.1. It is possible to notice in this case that the numerical solution is exact everywhere but in the single cell containing the non-classical shock. However, as already said, the value in this cell coincides with the average of the corresponding exact solution and using (3.6) we can recover the exact location of the discontinuity.

Case I: We consider Riemann type initial data with

$$
\rho_{0}(x)=\left\{\begin{array}{ll}
0.4 & \text { if } x<0.5, \\
0.5 & \text { if } x>0.5,
\end{array} \quad y_{0}=0.5 .\right.
$$

The solution is given by two classical shocks separated by a non-classical discontinuity, as illustrated in Figure 4.2, left.

Case II: We consider Riemann type initial data with

$$
\rho_{0}(x)=\left\{\begin{array}{ll}
0.8 & \text { if } x<0.5, \\
0.5 & \text { if } x>0.5,
\end{array} \quad y_{0}=0.5 .\right.
$$

The values of the initial conditions create a rarefaction wave followed by non-classical and classical shocks, as illustrated in Figure 4.2, right.

Case III: We consider the following initial data

$$
\rho_{0}(x)=\left\{\begin{array}{ll}
0.8 & \text { if } x<0.5, \\
0.4 & \text { if } x>0.5,
\end{array} \quad y_{0}=0.4 .\right.
$$

In this case, the bus initial position is not aligned with the discontinuity. We can see that the values of the initial conditions generate a rarefaction wave followed by nonclassical and classical shocks on the density that are created when the bus approaches the rarefaction and initiates a moving bottleneck, as illustrated in Figure 4.3.

Case IV: We consider the following initial data

$$
\rho_{0}(x)= \begin{cases}\hat{\rho}_{\alpha} & \text { if } x<0.25 \\ \check{\rho}_{\alpha} & \text { if } 0.25<x<0.5, \quad y_{0}=0.25 \\ 0.95 & \text { if } x>0.5\end{cases}
$$

The solution is given by a non-classical shock and a classical one that collide. After the collision a classical shock is generated, as illustrated in Figure 4.4.

For cases I and II we also show the convergence curves in Figure 4.5. It represents the $\log -\log \mathbf{L}^{\mathbf{1}}$ error between the numerical solution and the exact one versus mesh size. The numerical order of convergence can be found in Table 4.1. 


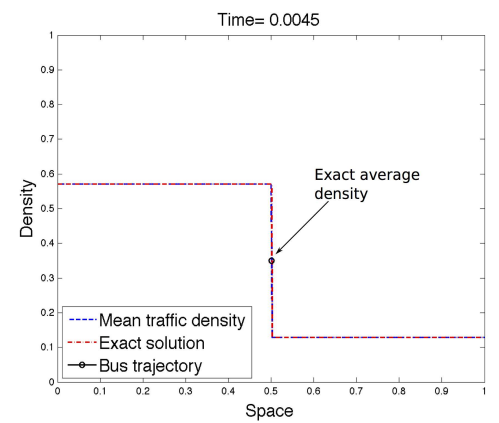

Fig. 4.1: Evolution in time of the density corresponding to initial data (4.1) (left) with $\Delta x=0.001$ compared with the exact solution.
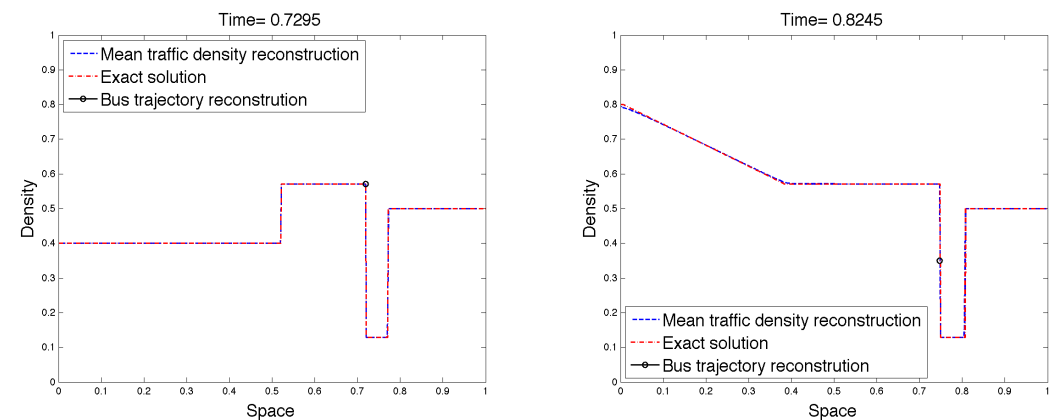

Fig. 4.2: Evolution in time of the density corresponding to initial data (4.2) (left) and (4.3) (right) with $\Delta x=0.001$ compared with the exact solution.

\begin{tabular}{|c|c|c|}
\hline$\Delta x$ & Order of convergence for Case I & Order of convergence for Case II \\
\hline 0.1 & 1.1762 & 0.8212 \\
\hline 0.05 & 0.9928 & 0.8794 \\
\hline 0.025 & 1.1360 & 0.9494 \\
\hline 0.0125 & 1.5980 & 1.4522 \\
\hline 0.00625 & 0.7769 & 1.0049 \\
\hline 0.003125 & 0.8473 & 1.0103 \\
\hline 0.0015625 & 0.8871 & 1.1898 \\
\hline
\end{tabular}

Table 4.1: Order of convergence for the reconstruction scheme, corresponding to initial data (4.2) and (4.3) .

5. Conclusions In this paper, we introduced a reconstruction based numerical method that is able to capture numerically non-classical shocks for a coupled PDEODE problem with moving constraints. The algorithm is conservative on a fixed mesh and isolated classical and non-classical shocks are propagated exactly. We showed several test cases, including shock interactions and tests that are more significant from a modeling point of view. The convergence of the method was shown numerically. 

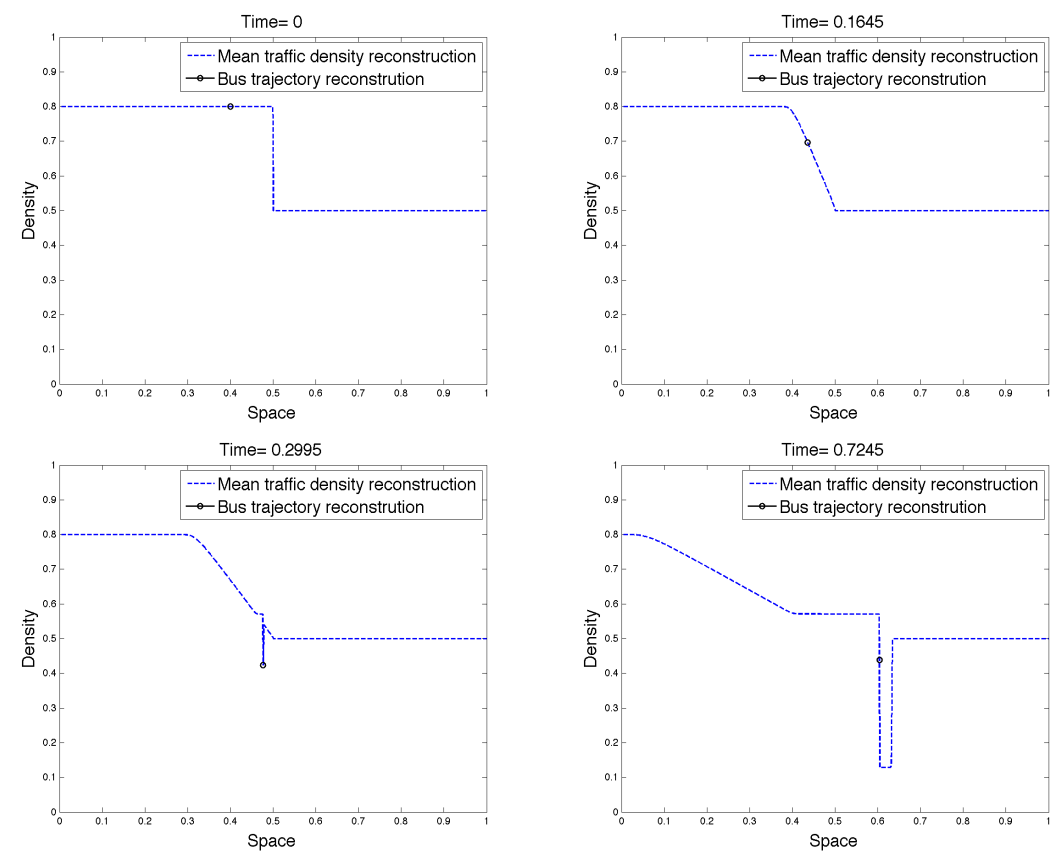

Fig. 4.3: Evolution in time of the density at different times corresponding to initial data (4.4) and $\Delta x=0.001$.
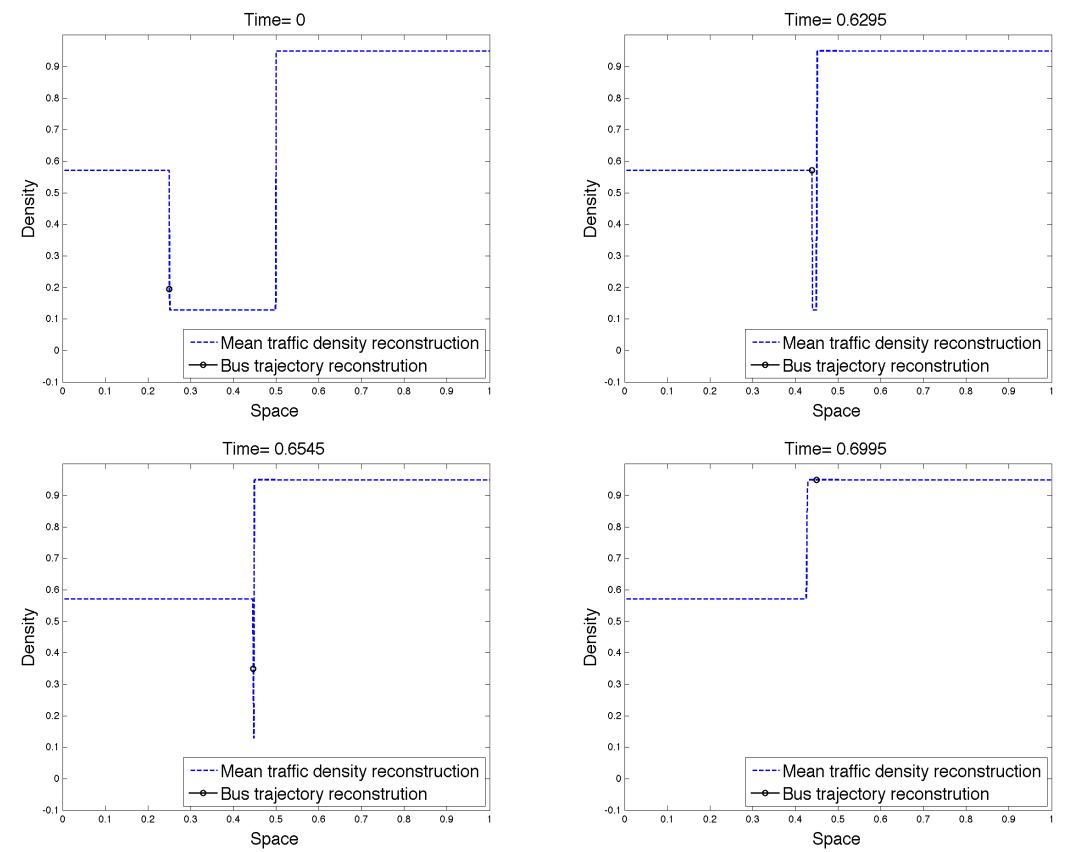

Fig. 4.4: Evolution in time of the density at different times corresponding to initial data (4.5) and $\Delta x=0.001$. 


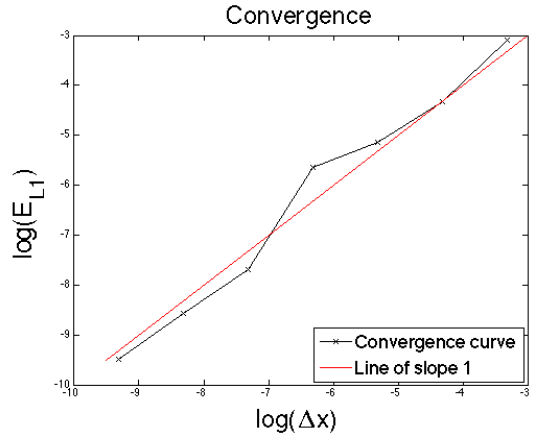

(a) Classical and non-classical shock - Case I

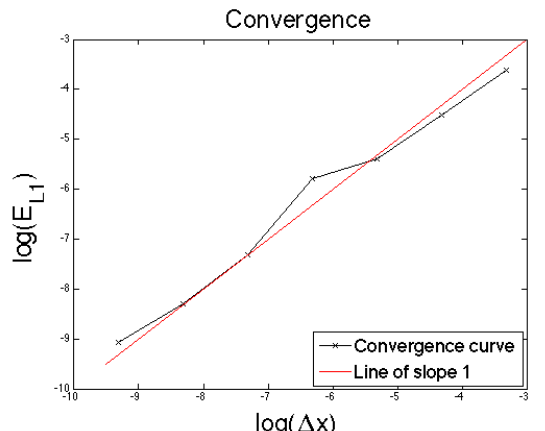

(b) Rarefaction and non-classical shock - Case II

Fig. 4.5: $\mathbf{L}^{\mathbf{1}}$ convergence.

\section{REFERENCES}

[1] B. Andreianov, P. Goatin, and N. Seguin. Finite volume schemes for locally constrained conservation laws. Numer. Math., 115(4):609-645, 2010. With supplementary material available online.

[2] R. Borsche, R. Colombo, and M. Garavello. Mixed systems: Odes - balance laws. Journal of Differential equations, 252:2311-2338, 2012.

[3] B. Boutin, C. Chalons, F. Lagoutière, and P. G. LeFloch. Convergent and conservative schemes for nonclassical solutions based on kinetic relations. I. Interfaces and Free Boundaries, 10(3):399-421, 2008.

[4] G. Bretti and B. Piccoli. A tracking algorithm for car paths on road networks. SIAM Journal on Applied Dynamical Systems, 7:510-531, 2008.

[5] C. Chalons, P. Goatin, and N. Seguin. General constrained conservation laws. application to pedestrian flow modeling. Netw. Heterog. Media, 8(2):433-463, 2013.

[6] R. M. Colombo and P. Goatin. A well posed conservation law with a variable unilateral constraint. J. Differential Equations, 234(2):654-675, 2007.

[7] C. Daganzo and J. A. Laval. Moving bottlenecks: A numerical method that converges in flows. Transportation Research Part B, 39:855-863, 2004.

[8] C. Daganzo and J. A. Laval. On the numerical treatement of moving bottlenecks. Transportation Research Part B, 39:31-46, 2005.

[9] M. L. Delle Monache and P. Goatin. A front tracking method for a strongly coupled PDE-ODE system with moving density constraints in traffic flow. Discrete Contin. Dyn. Syst. Ser. S, $7(3): 435-447,2014$.

[10] M. L. Delle Monache and P. Goatin. Scalar conservation laws with moving constraints arising in traffic flow modeling: an existence result. J. Differential Equations, 2014. DOI:10.1016/j.jde.2014.07.014.

[11] M. Garavello and P. Goatin. The Aw-Rascle traffic model with locally constrained flow. J. Math. Anal. Appl., 378(2):634-648, 2011.

[12] I. Gasser, C. Lattanzio, and A. Maurizi. Vehicular traffic flow dynamics on a bus route. Multiscale Model. Simul., 11(3):925-942, 2013.

[13] F. Giorgi. Prise en compte des transports en commun de surface dans la modélisation macroscopique de l'écoulement du trafic. PhD thesis, Institut National des Sciences Appliquées de Lyon, 2002.

[14] S. Godunov. A finite difference method for the numerical computation of discontinuous solutions of the equations of fluid dynamics. Matematicheskii Sbornik, 47:271-290, 1959.

[15] F. Lagoutière. Stability of reconstruction schemes for scalar hyperbolic conservation laws. Communication in mathematical sciences, 6(1):57-70, 2008.

[16] C. Lattanzio, A. Maurizi, and B. Piccoli. Moving bottlenecks in car traffic flow: a PDE-ODE coupled model. SIAM J. Math. Anal., 43(1):50-67, 2011.

[17] J.-P. Lebacque, J. B. Lesort, and F. Giorgi. Introducing buses into first-order macroscopic traffic flow models. Transportation Reasearch Record, 1644:70-79, 1998. 
[18] M. J. Lighthill and G. B. Whitham. On kinematic waves. II. A theory of traffic flow on long crowded roads. Proc. Roy. Soc. London Ser. A, 229:317-346, 1955.

[19] P. I. Richards. Shock waves on the highway. Operations Research, 4:42-51, 1956. 